\title{
Radiomic Nomogram: Pretreatment Evaluation of Local Recurrence in Nasopharyngeal Carcinoma based on MR Imaging
}

\author{
Lu Zhang1 ${ }^{*}$, Hongyu Zhou ${ }^{*}$, Dongsheng Gu2 ${ }^{*}$, Jie Tian²*, Bin Zhang3,4, Di Dong2, Xiaokai Mo1, Jing Liu ${ }^{5}$, \\ Xiaoning Luo ${ }^{1}$, Shufang Pei ${ }^{1}$, Yuhao Dong ${ }^{1}$, Wenhui Huang1, Qiuyin Chen ${ }^{3,4}$, Changhong Liang1, \\ Zhouyang Lian ${ }^{\circledR}$, Shuixing Zhang ${ }^{\boxplus}$ \\ 1. Department of Radiology, Guangdong Provincial People's Hospital/ Guangdong Academy of Medical Sciences, Guangzhou, Guangdong, PR China \\ 2. Institute of Automation, Chinese Academy of Sciences, CAS Key Laboratory of Molecular Imaging, Beijing, PR China \\ 3. Medical Imaging Center, First Affiliated Hospital of Jinan University, Guangzhou, Guangdong, PR China \\ 4. Institute of Molecular and Functional Imaging, Jinan University, Guangzhou, Guangdong, PR China \\ 5. Affiliated Hospital of Guizhou Medical University, Guiyang, Department of Radiology Guiyang, Guizhou, PR China \\ ${ }^{*}$ Lu Zhang, Hongyu Zhou, Dongsheng Gu, and Jie Tian contributed equally to this work.
}

$\triangle$ Corresponding authors: Shuixing Zhang and Zhouyang Lian. Department of Radiology, Guangdong Provincial People's Hospital/ Guangdong Academy of Medical Sciences, No. 106 Zhongshan Er Road, 510080 Guangzhou, Guangdong Prov., People's Republic of China. E-mail: zsx7515@jnu.edu.cn; Tel: +86 20 83870125; Fax: +86 2083870125

(c) The author(s). This is an open access article distributed under the terms of the Creative Commons Attribution License (https://creativecommons.org/licenses/by/4.0/). See http://ivyspring.com/terms for full terms and conditions.

Received: 2019.01.20; Accepted: 2019.05.25; Published: 2019.07.10

\begin{abstract}
Background: To develop and validate a radiomic nomogram incorporating radiomic features with clinical variables for individual local recurrence risk assessment in nasopharyngeal carcinoma (NPC) patients before initial treatment.

Methods: One hundred and forty patients were randomly divided into a training cohort $(\mathrm{n}=80)$ and a validation cohort $(n=60)$. A total of 970 radiomic features were extracted from pretreatment magnetic resonance (MR) images of NPC patients from May 2007 to December 2013. Univariate and multivariate analyses were used for selecting radiomic features associated with local recurrence, and multivariate analyses was used for building radiomic nomogram.

Results: Eight contrast-enhanced T1-weighted (CET1-w) image features and seven T2-weighted (T2-w) image features were selected to build a Cox proportional hazard model in the training cohort, respectively. The radiomic nomogram, which combined radiomic features and multiple clinical variables, had a good evaluation ability (C-index: 0.74 [95\% Cl: $0.58,0.85])$ in the validation cohort. The radiomic nomogram successfully categorized those patients into low- and high-risk groups with significant differences in the rate of local recurrence-free survival $(P<0.05)$.

Conclusions: This study demonstrates that MR imaging-based radiomics can be used as an aid tool for the evaluation of local recurrence, in order to develop tailored treatment targeting specific characteristics of individual patients.
\end{abstract}

Key words: Magnetic Resonance Imaging; Nasopharyngeal Carcinoma; Local Recurrence; Radiomic Feature; Nomogram

\section{Introduction}

Nasopharyngeal carcinoma (NPC) is a rare malignancy in most parts of the world, with incidence rate of approximately 1.1 cases per 100,000 people [1]. However, NPC is more common in South-Eastern Asia, particularly in southern China, with an annual incidence rate of 80 cases per 100,000 people [2-6]. Approximately $11 \%$ of NPC patients develop tumor recurrence at the primary or/and regional site after definitive therapy [7, 8]. With the use of intensity-modulated radiotherapy and concurrent 
chemoradiotherapy, the control of local recurrence has substantially improved $[9,10]$. However, only a portion of recurrent NPC patients have benefited from the improved treatment, which may be related to the specific characteristics of individuals $[1,9]$. The outcome of salvage treatment remains very poor [11, 12], with the survival after local recurrence equating to $<18.3$ months, and its management remains a difficult issue for clinicians $[13,14]$. It is therefore vital to define the reliable prognostic factors that could identify NPC patients at low- or high-risk of local recurrence.

Currently, magnetic resonance imaging (MRI) is a traditional and important tool for pretreatment staging and the determination of treatment programs for NPC [15, 16]. The traditional MRI, such as T1-weighted (T1-w) imaging and T2-weighted (T2-w) imaging, is mainly based on the anatomy of tumor invasion and does not consider intratumor heterogeneity so that they failed to evaluate the risk of individual patient based on specific intratumor characteristics [17, 18]. Many functional MRI sequences have been reported, such as diffusion-weighted imaging (DWI), diffusion tensor imaging (DTI), perfusion-weighted imaging (PWI), intravoxel incoherent motion (IVIM), could reflect intratumor features in different cancers $[19,20]$. However, they are not as regular sequences for NPC patients, because the inherent anatomic constraints could cause all kinds of artifacts. Recent studies have found that radiomics analysis, which is based on imaging data, could provide additional information reflecting the underlying intratumor heterogeneity [21, 22].

Radiomics, a rapidly emerging field, transforms medical images into mineable high-dimensional quantitative features via a large number of automatically extracted data-characterization algorithms [23, 24]. These numerous quantitative imaging features are extracted from entire tumors in different modalities (e.g. computed tomography [CT], MRI, or positron emission tomography-computed tomography [PET/CT]), and they may reflect intratumor heterogeneity which are closely associated with cancer staging, prognostic prediction, and response to treatment [25-27]. Identification of the intratumor heterogeneity of individual patients has the potential to yield important insights for targeted therapeutic selection and drug development [22, 28]. Several studies have observed the predictive ability of radiomic features in many cancers (e.g. head and neck squamous cell carcinoma [29], lung cancer [30], rectal cancer [31, 32], and breast cancer [33]), and revealed that radiomic features are associated with progression-free survival (PFS), recurrence, metastasis, and other clinical outcomes [34, 35]. Moreover, an intratumor heterogeneity survey for NPC patients has been assessed with regard to progression-free survival and treatment response [36-38]. These previous studies have indicated that radiomic features are an independent factor for the prediction of progression-free survival in advanced NPC patients. However, how radiomic features might differ between patients with different risk of local recurrence has not been established.

The aim of this study was to investigate whether radiomic features were associated with local recurrent NPC. Furthermore, we integrated radiomic features with clinical variables to build a nomogram for stratifying low- or high-risk local recurrent NPC patients.

\section{Materials and Methods}

\section{Patients}

This retrospective study was approved by the ethics committee of Guangdong General Hospital review board. All patients provided written informed consent. Our hospital database was searched to identify all patients with newly diagnosed NPC between May 2007 and December 2014 who were treated with a radiotherapy or chemoradiotherapy regimen. The study eligibility criteria were (a) patients with histologically-confirmed NPC; (b) patients who had not received treatment (surgery, radiotherapy, or chemoradiotherapy) before MRI scans; (c) patients who had undergone pre-treatment MRI scans for review (including CET1-w and T2-w images); and (d) patients who were followed-up every 1-3 months during the first 2 years, every 6 months in years 2-5, and annually thereafter. Patients with known magnetic resonance contraindications, insufficient follow-up data, any treatments (radiotherapy, chemotherapy, or chemoradiotherapy) before their first MRI scan, or a history of previous or synchronous malignant tumors, were excluded. The histological subtype of the patients' tumors was categorized according to the World Health Organization standards and included type I (differentiated keratinizing carcinoma), type II (differentiated non-keratinizing carcinoma), and type III (undifferentiated non-keratinizing carcinoma) [39]. Eligible patients were randomly divided into two cohorts at a ratio of 4:3. Eighty patients were assigned to the training cohort, while the remaining 60 patients were assigned to the independent validation cohort.

Baseline clinical variables were collected, including age, gender, $\mathrm{T}$ stage, $\mathrm{N}$ stage, histology, hemoglobin (HGB), and platelet count [40]. The primary endpoint of this study was local 
recurrence-free survival (LRFS), which was defined as the time from the start of the MR examination until the date of local recurrence $[12,13]$. The minimum follow-up time for patients without local recurrence was 36 months after the first MR examination. All local recurrences were diagnosed by flexible nasopharyngoscopy and biopsy and/or MRI scanning of the nasopharyngeal area and neck.

\section{Treatment}

The targets of radiation therapy (RT) are the nasopharynx and adjacent at-risk tissues, and both sides of the neck (levels $\mathrm{Ib}-\mathrm{V}$, and the retropharyngeal nodes) [1]. Patients received a cumulative radiation dose equivalent to 66 Gy or greater to the primary tumor, 60-66 Gy to the involved neck area, and 50 Gy or greater to the adjacent at-risk tissues [41]. Early stage patients (stage I-II) were treated with RT alone, while advanced stages (stage III-IV) were treated with RT and concurrent chemotherapy [26, 40]. The concurrent chemoradiotherapy regimen comprised of cisplatin $\left(40 \mathrm{mg} / \mathrm{m}^{2} 5\right.$ days per week, 6-7 cycles), beginning on the first day of radiotherapy.

\section{Radiomics workflow}

The radiomics workflow is presented in Fig. 1, including (1) image acquisition, (2) image segmentation, (3) feature extraction, (4) feature selection, and (5) model building.

\section{Image acquisition, image segmentation, and feature extraction}

All patients underwent non-contrast and contrast-enhanced nasopharyngeal and neck $1.5 \mathrm{~T}$ MRI scans (Signa EXCITE HD, TwinSpeed, GE Healthcare, Milwaukee, WI, USA). The acquisition parameters were as follows: axial T2-weighted spin-echo images (repetition time [TR]/echo time [TE]: 5000/85 ms, field of view [FOV] $=23 \times 23 \mathrm{~cm}$, number of excitations $[\mathrm{NEX}]=2.0$, slice thickness $=4$ $\mathrm{mm}$, spacing between slices $=1.0 \mathrm{~mm}$ ) and axial contrast- enhanced T1-weighted spin-echo images (TR/TE: $687 / 16 \mathrm{~ms}, \mathrm{FOV}=23 \times 23 \mathrm{~cm}, \mathrm{NEX}=2.0$, slice thickness $=4 \mathrm{~mm}$, spacing between slices $=1.0$ $\mathrm{mm})$. We included axial T2-w Digital Imaging and Communications in Medicine (DICOM) images and CET1-w DICOM images that had been archived using Picture Archiving and Communication Systems (PACS).

The segmentation for region-of-interest (ROI) settings was performed using ITK-SNAP software (open source software; https://itk.org/) All manual segmentations of the tumor were performed by two radiologist with 11 years of experience in head and neck MR image (O.Y. and B.G) in a blinded fashion, and each segmentation was validated by a senior radiologist with 15 years of experience in head and neck MR image (S.Z.). The ROI included the whole tumor and was delineated on both the axial T2-w and CET1-w images in each slice.

The feature extraction was performed in MATLAB R2014a (Mathworks, Natick, MA, USA) using our in-house algorithms designed to extract four different feature classes, including first-order statistics features, shape- and size-based features, statistics-based textural features, and wavelet features, which are described in detail in Supplementary Methods 1. DICOM files (MR images + tumor contours) were imported into the algorithms to extract the radiomic features. A total of 970 complete radiomic features were extracted from MR images, including 485 features from the CET1-w images and 485 features from the T2-w images.

\section{Feature selection and model building}

The recursive feature elimination with logistic regression algorithm (LR-RFE) was used to select the most predictive features with the highest area under the curve (AUC) (containing the first $\mathrm{n}$ features) [42, 43]. The RFE method continuously eliminates the features that did not significantly contribute to the model based on the iterative method, finally obtaining ranked features based on the number of iterations when the feature was discarded. With the ranked features, different feature sets could be obtained by selecting the top-n features from the ordered sequence $(1 \leq n \leq N)$. The set of first $n$ features was fed into the local recurrence classifier, and its performance for differentiating low- and high-risk of local recurrence in NPC could be evaluated by a receiver-operating characteristic (ROC) curve and AUC. Finally, a subset with the highest AUC (containing the first $\mathrm{n}$ features) was selected as the best feature subset for the discrimination task. The LR-RFE method was conducted in the training cohort and validated in the independent cohort. The LR-RFE algorithm was implemented in Python with the Sklearn package. After feature selection, we used Cox regression to build a model. The non-zero coefficient of the selected feature was defined as the radiomic score (Rad-score); the Rad-score was calculated for each patient using a formula derived from the selection features weighted by their regression coefficient. Radiomic features were built using the Rad-score. In this study, the CET1-w and $\mathrm{T} 2-\mathrm{w}$ images were used to establish the prediction model, and obtained the risk scores Rad-score1 and Rad-score2, respectively. Finally, we used cox regression analysis to determine the association of Rad-score, ${ }_{1}$ Rad-score, clinical $^{2}$ variables with local recurrence in the training cohort. A Rad-score was calculated for each patient derived 
from identified predictors weighted by their regression coefficient. We calculated the median Rad-score, which classified NPC patients into low-risk and high-risk groups.

\section{Validation of the radiomic nomogram}

The radiomic nomogram, which integrated the radiomic features and clinical variables, was built for prediction based on the Cox proportional hazards regression model. The radiomic nomogram performance was measured quantitatively using the concordance index (C-index) [44]. The concordance index and calibration curve were obtained from multivariable Cox proportional hazard regression analyses. The threshold of $\mathrm{p}$ value was $0.05(\mathrm{P}<0.05)$ for the "good" calibration in this analysis. Patients were stratified into high- or low-risk groups based on the Rad-score, the threshold of which was delimited by using the median Rad-score. Patients with median scores were placed in high-risk groups. We also used a survival analysis to measure the difference in the LRFS between high- and low- risk groups, which was assessed in the training cohort, and then confirmed in the validation cohort [45]. We also performed a cox regression analysis to test the significant of clinical variables alone, included age, gender, $\mathrm{T}$ stage, $\mathrm{N}$ stage, HGB, and platelet count.

\section{Statistical analysis}

The statistical analyses were conducted using $\mathrm{R}$ software, version 3.1.3 (http://www.R-project.org). We performed univariate and multivariate analyses in this study. The Kaplan-Meier and log-rank methods were used to analyze the univariate discrimination of the LRFS, which was grouped by models. Survival analysis and Kaplan-Meier survival curves were processed with the "survival" and "survcomp" packages. Multivariate logistic regression was conducted with the "rms" package. Cox proportional hazards regression, nomograms, and calibration curves were calculated by the rms package. C-index calculation was performed with the "Hmisc" package. The clinical characteristics of training and validation cohorts were compared by using an independent samples t-test, $X 2$ test, or Mann-Whitney $U$ test, as appropriate. We performed correlation analysis to evaluate relationship between clinical variables and local recurrence with the "stats" package. All statistical tests were two-sided, and $P$ values of $<0.05$ were considered significant.

\section{Results}

\section{Clinical characteristics}

A total of 140 patients with pathologically confirmed NPC were included in this study. The training and validation cohorts were similar in terms of baseline clinical variables $(P>0.05)$. The median LRFS time was 26 months (range: 3-68 months). The results of correlation analysis showed that N-stage is significant associated with local recurrence but other clinical variables are not significant. The patient characteristics are summarized in Table 1.

Table 1: Demographic and clinical characteristics of patients in the training cohort and validation cohort. Statistical comparison between the training cohort and the validation cohort was conducted with the Mann-Whitney $U$ test for continuous variables and the $\mathrm{X} 2$ test for categorical variables.

\begin{tabular}{|c|c|c|c|c|}
\hline Characteristic & Type & $\begin{array}{l}\text { Training Cohort } \\
(\%) \\
n=80\end{array}$ & $\begin{array}{l}\text { Validation cohort } \\
(\%) \\
n=60\end{array}$ & P-value \\
\hline \multirow[t]{2}{*}{ Gender } & Male & $54(67.5)$ & $49(81.7)$ & 0.06 \\
\hline & Female & $26(32.5)$ & 11 (18.3) & \\
\hline \multirow[t]{2}{*}{ Age (years) } & Range & $14-71$ & $14-67$ & 0.07 \\
\hline & Median \pm STD & $46 \pm 10$ & $42 \pm 12$ & \\
\hline \multirow[t]{4}{*}{ Overall stage } & I & $1(1.3)$ & $0(0)$ & 0.16 \\
\hline & II & $12(15)$ & $2(3.3)$ & \\
\hline & III & $46(57.5)$ & $42(70)$ & \\
\hline & IV & $21(26.2)$ & $16(26.7)$ & \\
\hline \multirow[t]{4}{*}{ T stage } & I & $6(7.5)$ & $5(8.3)$ & 0.08 \\
\hline & II & $30(37.5)$ & $11(18.3)$ & \\
\hline & III & $30(37.5)$ & $33(55)$ & \\
\hline & IV & $14(17.5)$ & $11(18.3)$ & \\
\hline \multirow[t]{4}{*}{ N stage } & I & $6(7.5)$ & $5(8.3)$ & 0.16 \\
\hline & II & $31(38.7)$ & $13(21.7)$ & \\
\hline & III & $36(45)$ & $37(61.7)$ & \\
\hline & IV & $7(8.8)$ & $5(8.3)$ & \\
\hline \multirow[t]{3}{*}{ Histology } & WHO type I & $0(0)$ & $0(0)$ & 0.73 \\
\hline & WHO type II & $2(2.5)$ & $1(1.7)$ & \\
\hline & WHO type & $78(97.5)$ & $59(98.3)$ & \\
\hline \multirow[t]{2}{*}{ Hemoglobin (g/L) } & $<136$ & $44(55)$ & $30(50)$ & 0.56 \\
\hline & $\geq 136$ & $36(45)$ & $30(50)$ & \\
\hline \multirow{2}{*}{$\begin{array}{l}\text { Platelet counts, } \\
\times 10^{9} / \mathrm{L}\end{array}$} & $<238$ & $47(58.8)$ & $26(43.3)$ & 0.07 \\
\hline & $\geq 238$ & $33(41.2)$ & $34(56.7)$ & \\
\hline
\end{tabular}

Radiomic feature extraction, feature selection, and model building

In total, the eight features selected from CET1-w images were as follows: CET1-w_1_GLCM_cluster_ shade, CET1-w_3_fos_median, CET1-w_3_fos_mean, CET1-w_4_fos_skewness, CET1-w_Surface_to_ volume_ratio, CET1-w_6_fos_skewness, CET1-w_4_ fos_mean, and CET1-w_4_fos_median. We used these features to establish a Cox proportional hazard model (CET1-w C-index 0.60 [95\% CI: 0.49-0.72]. Based on the coefficient of eight features, we calculated the Rad-score1 for CET1-w Cox (Supplementary Methods 3). Similarly, we selected seven features from the T2-w images as follows: T2-w_4_GLCM_cluster_shade, T2-w_6_GLCM_autocorrelation, T2-w_6_GLCM_ IMC1， T2-w_1_GLCM_cluster_shade, T2-w_7_fos_ mean, T2-w_1_GLRLM_LRHGLE, and T2-w_7_ GLCM_sum_average, and established a Cox proportional hazard model (T2-w C-index 0.62 [95\% 
CI: $0.52,0.71])$. The Rad-score2 of T2-w Cox was also calculated (Supplementary Methods 3).

Cox regression analysis determined that Rad-score $_{1}$, Rad-score, , gender, age, HGB, and $\mathrm{N}$-stage were associated with local recurrence in the training cohort. A Rad-score was calculated for each patient using the following formula derived from the above independent predictors weighted by their regression coefficient:

$$
\begin{gathered}
\text { Rad-score }=0.88663^{*} \text { Rad-score } 1+0.50748^{*} \text { Rad-score } \\
+0.02159^{*} \text { gender }+0.02012^{*} \text { age }+0.00691^{*} \mathrm{HGB} \\
-0.21954^{*} \mathrm{~N} \text {-stage }
\end{gathered}
$$

The contribution of the selected parameters with their coefficients for the predictive model construction is presented in the form of a histogram in Fig. 2. The model that incorporated the above independent predictors was developed and presented as the radiomic nomogram (Fig. 3A) and provided a C-index of 0.69 (95\% CI: 0.59-0.77). The calibration curves of the radiomic nomogram for the probability of local recurrence occurred at 2 or 3 years, as illustrated in Fig. 3B, and they exhibited better agreement between the estimation with the radiomic nomogram and actual observation.

\section{Validation of the radiomic nomogram}

Using the second radiomic features to similarly extract and select 60 patients as the internal validation cohort, the radiomic nomogram yielded a C-index of 0.74 (95\% CI: 0.58-0.85). Good calibration was observed for the probability of local recurrence in the validation cohort (Figure not provided). We also built a clinical model based on the clinical variables. After cox regression analysis, only $\mathrm{N}$ stage was remained as independent predictors of local recurrence. The clinical model yielded a C-index of 0.56 (95\% CI, $0.66-0.45)$ in the training cohort and $0.59(95 \% \mathrm{CI}$, $0.68-0.49)$ in the validation cohort. The radiomic nomogram exhibited better prediction performance than the radiomic features and clinical variables alone, in both the training cohort and the validation cohort.

The patients were categorized into low- and high-risk groups based on the median Rad-score. Patients in the low-risk group (Rad-score $<5.50$ ) were significant longer LRFS than did patients in the high-risk group (Rad-score $\geq 5.50$ ) (Fig. 4, $P=0.008$ ).

\section{Discussion}

Using radiomics-based feature analysis, we found that radiomic features were closely association with local recurrence in NPC patients. In this study, we built a radiomic nomogram that combined the radiomic features and clinical variables to evaluate the risk of local recurrence in NPC patients before initial treatment, and classified NPC patients into high risk and low risk groups. The radiomic nomogram provides a visual tool for optimal clinical decisions, enabling clinicians to perform inexpensive, earlier identification of NPC patients at high risk of local recurrence.

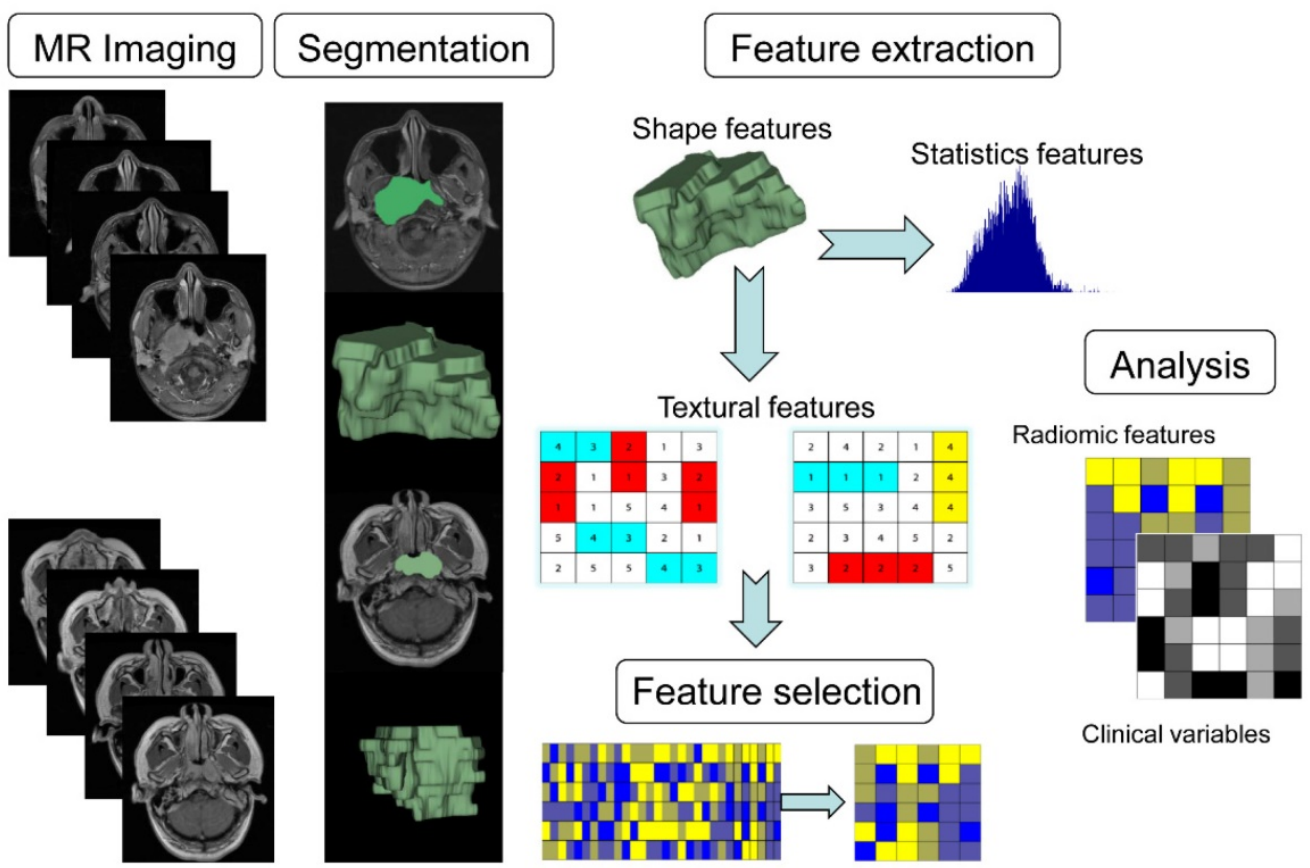

Figure 1: Workflow of the radiomics analysis. (1) Acquisition of high-quality magnetic resonance images. (2) Segmented region of interest (ROI) that contains either the whole tumor rendered in three dimensions. (3) Quantitative features were extracted. (4) Robust features were selected. (5) A combined model was built that integrated the radiomic features and multiple clinical variables. 


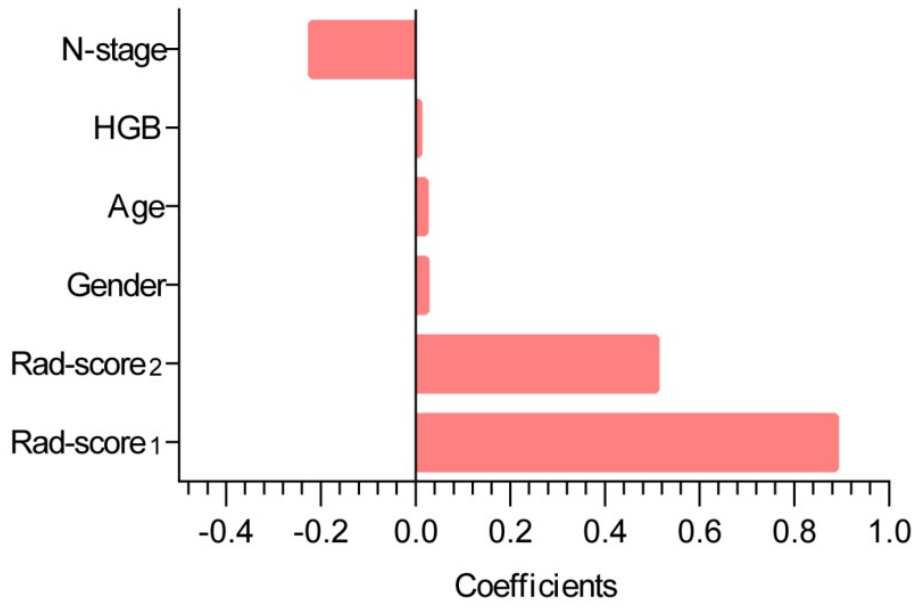

Figure 2: Histogram illustrating the role of the selected parameters that contributed to the radiomic nomogram. The selected parameters are plotted on the $y$-axis with their coefficients in the Cox analysis plotted on the x-axis.
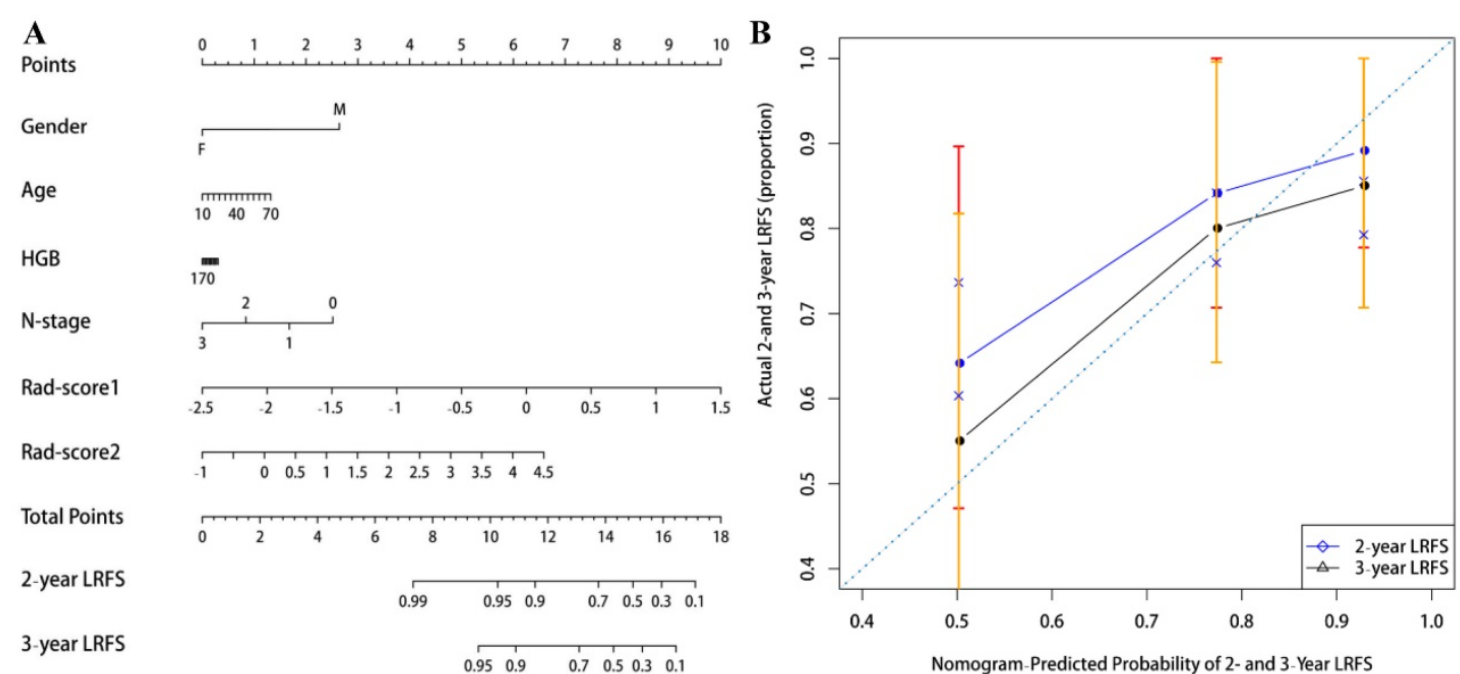

Figure 3: (A) A radiomic nomogram incorporated the Rad-scorel, Rad-score2, and clinical variables in the training cohort. (B) Calibration curve of local recurrence free survival probabilities at 2-years (blue line) and 3-years (black line) in nasopharyngeal carcinoma patients. The diagonal dotted line represents an ideal evaluation, while the blue and black solid lines represent the performance of the radiomic nomogram. The closer the fit to the diagonal dotted line, the better the evaluation.

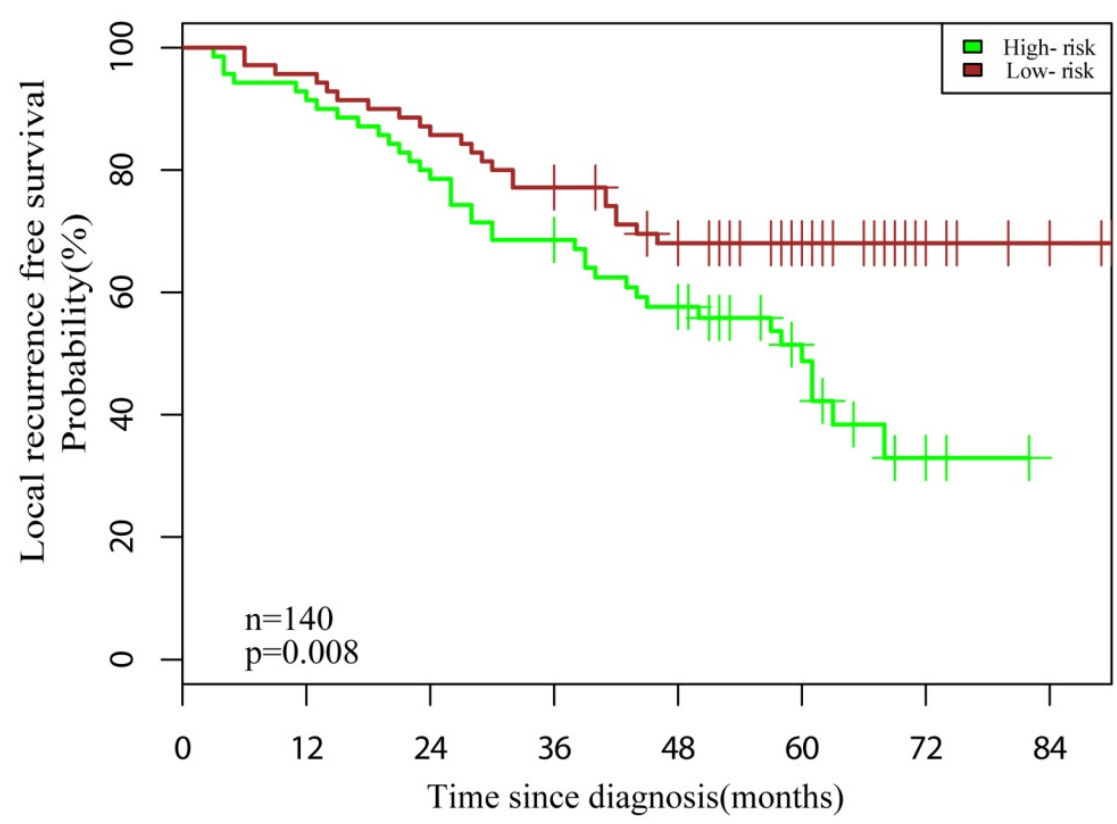

Figure 4: Graphs indicating the results of the Kaplan-Meier survival analyses according to the radiomic features to predict local recurrence-free probability in all datasets. A significant local recurrence free survival difference was noted between patients in the low- and high-risk groups $($ dataset, $n=140$, log-rank test, $P=0.008)$. 
Previous models of NPC demonstrated that MRI-based radiomic features were predictive in progression-free survival and treatment response without offering an evaluation of local recurrence [36, 46]. In this study, we found that radiomic features had a closely association with local recurrence in NPC patients, and the predictive performance of the nomogram model underwent significant improvement after the addition of radiomic features. The results can be explained by the fact that the quantitative image features were extracted from entire tumors, and hence they are likely to reflect the intra-tumor heterogeneity. Kwan et al [47] and Vallieres et al [48] have also supported that radiomic features was associated with distant metastasis and could reflect the characteristics of intratumor heterogeneity. The result also demonstrated that the contribution of the CET1-w images to the nomogram was slightly greater than that of the T2-w images. The result may indicate that the $\mathrm{T} 2-\mathrm{w}$ images mainly reflected tumor density and obscure boundaries, while the CET1-w images reflected intra-tumor heterogeneity and architecture (e.g., tumor angiogenesis) [49]. This previous finding of angiogenesis associated with tumor invasion lends credence to the possibility of CET1-w image differences possibly reflecting angiogenesis [41].

Age, gender, T-stage, N-stage, hemoglobin, platelet counts have been identified and evaluated in previous studies [50, 51]. In this study, after multivariate analyses, age, gender, N-stage, HGB were remained and combined with radiomic features to build a model for the evaluation of local recurrence in NPC patients. Our results indicated that N-stage was negatively associated with local recurrence in NPC patients, while previous findings supported that $\mathrm{N}$-stage was positive association with distant metastasis in NPC patients [52]. To validate the relationship between $\mathrm{N}$-stage and local recurrence, we conducted a correlation analysis and the result indicated that $\mathrm{N}$-stage was significantly associated with local recurrence. The possible reasons underlying the negative correlation of $\mathrm{N}$-stage are as follows: first, N-stage was based on the site of adenopathy and that of the greatest diameter [1]. However, occult micrometastasis in lymph nodes could exist regardless of the size [53]. Second, the irradiation area of the neck is unfixed compared with other head and neck irradiation area by using a thermoplastic head and shoulder mask. Therefore, the cervical lymph node could not receive enough dosing, which may result in local recurrence. Finally, we kept $\mathrm{N}$-stage as an important factor in the nomogram. In addition, age, gender and HGB were remained after filtration. Although these clinical variables provided slightly predictive strength to improve reclassification performance in this study, existing studies have demonstrated that they could serve as important markers of prognosis in NPC patients; therefore, we kept them as candidate factors in the nomogram [10, $50,54,55]$.

Several previous studies have made great efforts to build nomograms for individual local recurrence risk assessment in NPC patients based on clinical variables, such as Chen et al [56], which included age, the neutrophil/leukocyte ratio (NWR), pathological type, primary gross tumor volume, maxillary sinus invasion, ethmoidal sinus, invasion and lacerated foramen invasion. Wang et al [57] also reported that cervical lymph nodes $(\geq 3 \mathrm{~cm})$ were a risk factor for local recurrence in NPC patients treated with intensity-modulated radiotherapy. However, new biomarker that could reflect intratumor heterogeneity still need to be found to guide individual treatment for patients. The radiomic nomogram we developed in this study combined the clinical variables and the specific radiomic features, which selected from first-order statistics, shape descriptors, and texture features, could reflect intratumor heterogeneity. Our results also showed that the radiomic nomogram provides better predictive performance compared with radiomic features and clinical variables alone, as it refers to the combination between multiple clinical manifestations and intratumor heterogeneity. In additional, the radiomic nomogram could serve as both a scoring system and a visualized prediction tool, which could help physicians rapidly evaluate a patient with his/her expected LRFS via a simple calculation in clinical practice. Quantifying both of these risks is critical to the clinical decision of whether patients should receive any specific therapy and, if so, whether it should be less or more "intense." Notedly, we still need further prospective clinical validation before this model was used for clinic.

There were some limitations in the present analysis. First, this study was performed in an endemic area, single hospital and lacked external validation. Multicenter prospective validation in both NPC-endemic and nonendemic areas with a larger sample size is needed to acquire powerful evidence for clinical application. Second, the algorithms of radiomic model and statistical analysis were relatively unfamiliar and complicated for clinic. There will be a website or application to solve this problem, which doctors could get the results by uploading images and clinical variables.

In conclusion, we developed a radiomic nomogram integrated radiomic features and multiple clinical variables to predict and evaluate local recurrence in NPC patients. The radiomic nomogram 
classified NPC patients into high-risk and low-risk groups with significant difference in local recurrence-free survival, and it served as a visual tool to identify high-risk individuals who would benefit from aggressive therapeutic strategies.

\section{Abbreviations}

NPC: Nasopharyngeal carcinoma; MRI: Magnetic resonance imaging; CET1-w: Contrastenhanced T1-weighted image; T2-w: T2-weighted image; HGB: Hemoglobin; DICOM: Digital Imaging and Communications in Medicine; PACS: Picture Archiving and Communication Systems; ROI: Region-of-interest; AUC: Area under the curve; ROC: Receiver-operating characteristic.

\section{Supplementary Material}

Supplementary methods.

http://www.jcancer.org/v10p4217s1.pdf

\section{Acknowledgements}

We thank the anonymous reviewers for their insightful comments and great efforts to improve this manuscript.

\section{Financial Support}

We acknowledge financial support from the National Natural Science Foundation of China (81571664, 81871323, 81801665); the National Natural Science Foundation of Guangdong Province (2018B030311024); the Scientific Research General Project of Guangzhou Science Technology and Innovation Commission (201707010328); and the China Postdoctoral Science Foundation (2016M600145).

\section{Competing Interests}

The authors have declared that no competing interest exists.

\section{References}

1. Chan AT, Gregoire V, Lefebvre JL, Licitra L, Hui EP, Leung SF, et al. Nasopharyngeal cancer: EHNS-ESMO-ESTRO Clinical Practice Guidelines for diagnosis, treatment and follow-up. Annals of oncology : official journal of the European Society for Medical Oncology. 2012; 23 Suppl 7: vii83-5.

2. Torre LA, Bray F, Siegel RL, Ferlay J, Lortet-Tieulent J, Jemal A. Global cancer statistics, 2012. CA: a cancer journal for clinicians. 2015; 65: 87-108.

3. de Martel C, Ferlay J, Franceschi S, Vignat J, Bray F, Forman D, et al. Global burden of cancers attributable to infections in 2008: a review and synthetic analysis. The Lancet Oncology. 2012; 13: 607-15.

4. Su WH, Chiu CC, Yao Shugart $\mathrm{Y}$. Heterogeneity revealed through meta-analysis might link geographical differences with nasopharyngeal carcinoma incidence in Han Chinese populations. BMC cancer. 2015; 15: 598.

5. Kamran SC, Riaz N, Lee N. Nasopharyngeal carcinoma. Surgical oncology clinics of North America. 2015; 24: 547-61.

6. He S, Wang Y, Peng H, Yang L, Chen H, Liang S, et al. Pretreatment Alkaline Phosphatase and Epstein-Barr Virus DNA Predict Poor Prognosis and Response to Salvage Radiotherapy in Patients with Nasopharyngeal Carcinoma and Metachronous Bone-Only Metastasis. Journal of Cancer. 2017; 8: 417-24.

7. You R, Zou X, Wang SL, Jiang R, Tang LQ, Zhang WD, et al. New surgical staging system for patients with recurrent nasopharyngeal carcinoma based on the AJCC/UICC rTNM classification system. European journal of cancer (Oxford, England : 1990). 2015; 51: 1771-9.

8. Cui Y, Zhang C, Li X, Liu H, Yin B, Xu T, et al. Intravoxel Incoherent Motion Diffusion-weighted Magnetic Resonance Imaging for Monitoring the Early Response to ZD6474 from Nasopharyngeal Carcinoma in Nude Mouse. Scientific reports. 2015; 5: 16389.

9. Sun X, Su S, Chen C, Han F, Zhao C, Xiao W, et al. Long-term outcomes of intensity-modulated radiotherapy for 868 patients with nasopharyngeal carcinoma: an analysis of survival and treatment toxicities. Radiotherapy and oncology : journal of the European Society for Therapeutic Radiology and Oncology. 2014; 110: 398-403.

10. Tang XR, Li YQ, Liang SB, Jiang W, Liu F, Ge WX, et al. Development and validation of a gene expression-based signature to predict distant metastasis in locoregionally advanced nasopharyngeal carcinoma: a retrospective, multicentre, cohort study. The Lancet Oncology. 2018; 19: 382-93.

11. Blanchard P, Lee A, Marguet S, Leclercq J, Ng WT, Ma J, et al. Chemotherapy and radiotherapy in nasopharyngeal carcinoma: an update of the MAC-NPC meta-analysis. The Lancet Oncology. 2015; 16: 645-55.

12. Tian YM, Tian YH, Zeng L, Liu S, Guan Y, Lu TX, et al. Prognostic model for survival of local recurrent nasopharyngeal carcinoma with intensity-modulated radiotherapy. British journal of cancer. 2014; 110: 297-303.

13. Kong L, Wang L, Shen C, Hu C, Wang L, Lu JJ. Salvage Intensity-Modulated Radiation Therapy (IMRT) for Locally Recurrent Nasopharyngeal Cancer after Definitive IMRT: A Novel Scenario of the Modern Era. Scientific reports. 2016; 6: 32883 .

14. Chua ML, Chan AT. Gemcitabine: a game changer in nasopharyngeal carcinoma. Lancet (London, England). 2016; 388: 1853-4.

15. Mao YP, Xie FY, Liu LZ, Sun Y, Li L, Tang LL, et al. Re-evaluation of 6th edition of AJCC staging system for nasopharyngeal carcinoma and proposed improvement based on magnetic resonance imaging. International journal of radiation oncology, biology, physics. 2009; 73: 1326-34

16. Chen L, Hu CS, Chen XZ, Hu GQ, Cheng ZB, Sun Y, et al. Concurrent chemoradiotherapy plus adjuvant chemotherapy versus concurrent chemoradiotherapy alone in patients with locoregionally advanced nasopharyngeal carcinoma: a phase 3 multicentre randomised controlled trial. The Lancet Oncology. 2012; 13: 163-71.

17. Chicklore S, Goh V, Siddique M, Roy A, Marsden PK, Cook GJ. Quantifying tumour heterogeneity in 18F-FDG PET/CT imaging by texture analysis. European journal of nuclear medicine and molecular imaging. 2013; 40: 133-40.

18. Lian C, Ruan S, Denoeux T, Jardin F, Vera P. Selecting radiomic features from FDG-PET images for cancer treatment outcome prediction. Medical image analysis. 2016; 32: 257-68.

19. Fathi Kazerooni A, Nabil M, Zeinali Zadeh M, Firouznia K, Azmoudeh-Ardalan F, Frangi AF, et al. Characterization of active and infiltrative tumorous subregions from normal tissue in brain gliomas using multiparametric MRI. J Magn Reson Imaging. 2018; 48: 938-50.

20. Yuan Q, Kapur P, Zhang Y, Xi Y, Carvo I, Signoretti S, et al. Intratumor Heterogeneity of Perfusion and Diffusion in Clear-Cell Renal Cell Carcinoma: Correlation With Tumor Cellularity. Clinical genitourinary cancer. 2016; 14: e585-e94.

21. Zhang B, He X, Ouyang F, Gu D, Dong Y, Zhang L, et al. Radiomic machine-learning classifiers for prognostic biomarkers of advanced nasopharyngeal carcinoma. Cancer letters. 2017; 403: 21-7.

22. Lambin P, Leijenaar RTH, Deist TM, Peerlings J, de Jong EEC, van Timmeren J, et al. Radiomics: the bridge between medical imaging and personalized medicine. Nature reviews Clinical oncology. 2017; 14: 749-62.

23. Gillies RJ, Kinahan PE, Hricak H. Radiomics: Images Are More than Pictures, They Are Data. Radiology. 2016; 278: 563-77.

24. Aerts HJ, Velazquez ER, Leijenaar RT, Parmar C, Grossmann P, Carvalho S, et al. Decoding tumour phenotype by noninvasive imaging using a quantitative radiomics approach. Nature communications. 2014; 5: 4006.

25. Shen WC, Chen SW, Liang JA, Hsieh TC, Yen $\mathrm{KY}$, Kao $\mathrm{CH}$. [18]Fluorodeoxyglucose Positron Emission Tomography for the Textural Features of Cervical Cancer Associated with Lymph Node Metastasis and Histological Type. European journal of nuclear medicine and molecular imaging. 2017; 44: 1721-31.

26. Wu S, Zheng J, Li Y, Yu H, Shi S, Xie W, et al. A Radiomics Nomogram for the Preoperative Prediction of Lymph Node Metastasis in Bladder Cancer. Clinical cancer research : an official journal of the American Association for Cancer Research. 2017; 23: 6904-11.

27. Sala E, Mema E, Himoto Y, Veeraraghavan H, Brenton JD, Snyder A, et al. Unravelling tumour heterogeneity using next-generation imaging: radiomics, radiogenomics, and habitat imaging. Clinical radiology. 2017; 72: 3-10.

28. Gerlinger M, Rowan AJ, Horswell S, Larkin J, Endesfelder D, Gronroos E, et al. Intratumor heterogeneity and branched evolution revealed by multiregion sequencing. The New England journal of medicine. 2012; 366: 883-92.

29. Bogowicz M, Riesterer O, Ikenberg K, Stieb S, Moch H, Studer G, et al. Computed Tomography Radiomics Predicts HPV Status and Local Tumor Control After Definitive Radiochemotherapy in Head and Neck Squamous Cell Carcinoma. International journal of radiation oncology, biology, physics. 2017; 99: 921-8.

30. Buch K, Fujita A, Li B, Kawashima Y, Qureshi MM, Sakai O. Using Texture Analysis to Determine Human Papillomavirus Status of Oropharyngeal Squamous Cell Carcinomas on CT. AJNR American journal of neuroradiology. 2015; 36: 1343-8. 
31. Song J, Shi J, Dong D, Fang M, Zhong W, Wang K, et al. A New Approach to Predict Progression-free Survival in Stage IV EGFR-mutant NSCLC Patients with EGFR-TKI Therapy. Clin Cancer Res. 2018; 24: 3583-92.

32. Liu Z, Zhang XY, Shi YJ, Wang L, Zhu HT, Tang Z, et al. Radiomics Analysis for Evaluation of Pathological Complete Response to Neoadjuvant Chemoradiotherapy in Locally Advanced Rectal Cancer. Clinical cancer research : an official journal of the American Association for Cancer Research. 2017; 23: 7253-62.

33. Li H, Zhu Y, Burnside ES, Drukker K, Hoadley KA, Fan C, et al. MR Imaging Radiomics Signatures for Predicting the Risk of Breast Cancer Recurrence as Given by Research Versions of MammaPrint, Oncotype DX, and PAM50 Gene Assays. Radiology. 2016; 281: 382-91.

34. Peng J, Zhang J, Zhang Q, Xu Y, Zhou J, Liu L. A radiomics nomogram for preoperative prediction of microvascular invasion risk in hepatitis $B$ virus-related hepatocellular carcinoma. Diagnostic and interventional radiology (Ankara, Turkey). 2018; 24: 121-7.

35. Wu S, Zheng J, Li Y, Wu Z, Shi S, Huang M, et al. Development and Validation of an MRI-Based Radiomics Signature for the Preoperative Prediction of Lymph Node Metastasis in Bladder Cancer. EBioMedicine. 2018; 34: 76-84.

36. Zhang B, Tian J, Dong D, Gu D, Dong Y, Zhang L, et al. Radiomics Features of Multiparametric MRI as Novel Prognostic Factors in Advanced Nasopharyngeal Carcinoma. Clinical cancer research : an official journal of the American Association for Cancer Research. 2017; 23: 4259-69.

37. Liu J, Mao Y, Li Z, Zhang D, Zhang Z, Hao S, et al. Use of texture analysis based on contrast-enhanced MRI to predict treatment response to chemoradiotherapy in nasopharyngeal carcinoma. Journal of magnetic resonance imaging : JMRI. 2016; 44: 445-55.

38. Ji GW, Zhang YD. Biliary Tract Cancer at CT: A Radiomics-based Model to Predict Lymph Node Metastasis and Survival Outcomes. Radiology. 2019; 290: 90-8.

39. Ou SH, Zell JA, Ziogas A, Anton-Culver H. Epidemiology of nasopharyngeal carcinoma in the United States: improved survival of Chinese patients within the keratinizing squamous cell carcinoma histology. Annals of oncology: official journal of the European Society for Medical Oncology. 2007; 18: 29-35.

40. Edge SB, Compton CC. The American Joint Committee on Cancer: the 7th edition of the AJCC cancer staging manual and the future of TNM. Annals of surgical oncology. 2010; 17: 1471-4.

41. Xiao $W, X u$ A, Han $F$, Lin $X$, Lu, Shen $G$, et al Positron emission tomography-computed tomography before treatment is highly prognostic of distant metastasis in nasopharyngeal carcinoma patients after intensity-modulated radiotherapy treatment: a prospective study with long-term follow-up. Oral oncology. 2015; 51: 363-9.

42. Yang Z, Zhuan B, Yan Y, Jiang S, Wang T. Identification of gene markers in the development of smoking-induced lung cancer. Gene. 2016; 576: 451-7.

43. Zhu J, Hastie T. Classification of gene microarrays by penalized logistic regression. Biostatistics (Oxford, England). 2004; 5: 427-43.

44. Harrell FE, Jr., Califf RM, Pryor DB, Lee KL, Rosati RA. Evaluating the yield of medical tests. Jama. 1982; 247: 2543-6.

45. Schroder MS, Culhane AC, Quackenbush J, Haibe-Kains B. survcomp: an $\mathrm{R} /$ Bioconductor package for performance assessment and comparison of survival models. Bioinformatics (Oxford, England). 2011; 27: 3206-8.

46. Wang G, He L, Yuan C, Huang Y, Liu Z, Liang C. Pretreatment MR imaging radiomics signatures for response prediction to induction chemotherapy in patients with nasopharyngeal carcinoma. European journal of radiology. 2018; 98: 100-6.

47. Kwan JYY, Su J, Huang SH, Ghoraie LS, Xu W, Chan B, et al. Radiomic Biomarkers to Refine Risk Models for Distant Metastasis in HPV-related Oropharyngeal Carcinoma. International journal of radiation oncology, biology, physics. 2018; 102: 1107-16.

48. Vallieres M, Freeman CR, Skamene SR, El Naqa I. A radiomics model from joint FDG-PET and MRI texture features for the prediction of lung metastases in soft-tissue sarcomas of the extremities. Physics in medicine and biology. 2015; 60: 5471-96.

49. Ibrahim MA, Dublin AB. Magnetic Resonance Imaging (MRI), Gadolinium. StatPearls. Treasure Island, USA: StatPearls Publishing LLC; 2018

50. Li XH, Chang $\mathrm{H}, \mathrm{Xu} \mathrm{BQ}$, Tao YL, Gao J, Chen C, et al. An inflammatory biomarker-based nomogram to predict prognosis of patients with nasopharyngeal carcinoma: an analysis of a prospective study. Cancer medicine. 2017; 6: 310-9.

51. Liang W, Shen G, Zhang Y, Chen G, Wu X, Li Y, et al. Development and validation of a nomogram for predicting the survival of patients with non-metastatic nasopharyngeal carcinoma after curative treatment. Chinese journal of cancer. 2016; 35: 98

52. Xia WX, Zhang HB, Shi JL, Lu X, Wang L, Ye YF, et al. A prognostic model predicts the risk of distant metastasis and death for patients with nasopharyngeal carcinoma based on pre-treatment serum C-reactive protein and N-classification. European journal of cancer (Oxford, England : 1990). 2013; 49: 2152-60.

53. Dong $\mathrm{Y}$, Feng $\mathrm{Q}$, Yang $\mathrm{W}$, Lu $\mathrm{Z}$, Deng $\mathrm{C}$, Zhang $\mathrm{L}$, et al. Preoperative prediction of sentinel lymph node metastasis in breast cancer based on radiomics of T2-weighted fat-suppression and diffusion-weighted MRI. Eur Radiol. 2018; 28: 582-91.

54. Gao J, Tao YL, Li G, Yi W, Xia YF. Involvement of difference in decrease of hemoglobin level in poor prognosis of Stage I and II nasopharyngeal carcinoma: implication in outcome of radiotherapy. International journal of radiation oncology, biology, physics. 2012; 82: 1471-8

55. Chang H, Gao J, Xu BQ, Guo SP, Lu RB, Li G, et al. Haemoglobin, neutrophil to lymphocyte ratio and platelet count improve prognosis prediction of the TNM staging system in nasopharyngeal carcinoma: development and validation in 3,237 patients from a single institution. Clinical oncology (Royal College of Radiologists (Great Britain)). 2013; 25: 639-46.

56. Chen FP, Lin L, Qi ZY, Zhou GQ, Guo R, Hu J, et al. Pretreatment Nomograms for Local and Regional Recurrence after Radical Radiation Therapy for Primary Nasopharyngeal Carcinoma. Journal of Cancer. 2017; 8: 2595-603.

57. Wang L, Guo Y, Xu J, Chen Z, Jiang X, Zhang L, et al. Clinical Analysis of Recurrence Patterns in Patients With Nasopharyngeal Carcinoma Treated With Intensity-Modulated Radiotherapy. The Annals of otology, rhinology, and laryngology. 2017; 126: 789-97. 